Das Vorhandensein stärkerer $(p-d) \pi$-Anteile in der Sn-P-Bindung der $\left(\mathrm{R}_{3} \mathrm{Sn}\right)_{3} \mathrm{P}$-Verbindung wurde auch aus dem chemischen Verhalten gefolgert ${ }^{6}$. So war es z. B. nicht möglich, das Phosphoratom durch Anlagerung von Sauerstoff, Schwefel, Methyljodid oder $\mathrm{C}_{6} \mathrm{H}_{5} \mathrm{~N}-$ zu quaternisieren. Man muß daher annehmen, daß zumindest im Falle der Zinn-Verbindung auf Grund der Größenverhältnisse der d-Orbitale des Zinnatoms trotz der nichtebenen Struktur eine Überlappung mit dem freien Elektronenpaar des Phosphoratoms möglich ist.

In der Reihe $\left[\left(\mathrm{C}_{6} \mathrm{H}_{5}\right)_{3} \mathrm{Sn}\right]_{3} \mathrm{P},\left[\left(\mathrm{C}_{6} \mathrm{H}_{5}\right)_{3} \mathrm{Sn}\right]_{2} \mathrm{PC}_{6} \mathrm{H}_{5}$, $\left(\mathrm{C}_{6} \mathrm{H}_{5}\right)_{3} \mathrm{SnP}\left(\mathrm{C}_{6} \mathrm{H}_{5}\right)_{2}, \mathrm{P}\left(\mathrm{C}_{6} \mathrm{H}_{5}\right)_{3}(\delta=8 \mathrm{ppm})$ fällt auf, daß sich die Anderung der chemischen Verschie-

${ }^{6}$ H. Schumann, P. Schwabe u. A. Roth, unveröffentlicht. bung nicht durch feste Inkremente für die $\left(\mathrm{C}_{6} \mathrm{H}_{5}\right)_{3} \mathrm{Sn}$ Gruppe darstellen lassen, wie das in vielen Fällen für Verbindungen des Phosphors der Koordinationszahl 3 beobachtet wird ${ }^{7}$. So lassen sich z. B. die $\delta$-Werte der einzelnen Glieder der analogen Verbindungen $\left(\mathrm{CH}_{3}\right)_{\mathrm{n}} \mathrm{P}\left(\mathrm{C}_{6} \mathrm{H}_{5}\right)_{3-\mathrm{n}}(n=1,2,3)$ aus der Summe der von $F_{\text {LUCK }}$ angegebenen Substituenteninkremente ${ }^{8}$ mit guter Genauigkeit berechnen. Daraus läßt sich ebenfalls ableiten, daß die chemischen Verschiebungen nicht nur durch eine stetige Änderung des $\sigma$-Bindungsgerüstes bestimmt werden, sondern, daß offensichtlich andere, nicht additive Effekte eine Rolle spielen, die sehr wahrscheinlich mit den oben diskutierten $(\mathrm{p} \rightarrow \mathrm{d}) \pi$-Anteilen im Zusammenhang stehen.

7 E. F ${ }_{\text {LUCK }}$, Z. Naturforschg. 19 b, 869 [1964].

8 E. F ${ }_{\text {LUCK, }}$ Chem. Ber. 98, 2674 [1965].

\section{Zur Analyse des Kernresonanzspektrums des Cyan-allens}

Detlef Wendisch

Farbenfabriken Bayer AG, Leverkusen, IngenieurAbteilung AP

(Z. Naturforschg. 22 b, 353-354 [1967] ; eingeg. am 13. Januar 1967)

RedDy und Mitarbb. ${ }^{1}$ schätzten durch Analogiebetrachtungen die chemische Verschiebung des $\alpha$-Protons des Cyan-allens (I) zu $-200 \mathrm{~Hz}$ (relativ zu TMS für $40 \mathrm{MHz}$ ) ab. Walz und Kurtz ${ }^{2}$ untersuchten das Kernresonanzspektrum von reinem Cyan-allen bei $60 \mathrm{MHz}$ in verschiedenen Lösungsmitteln. Die geringe Aufspaltung des vorhandenen einzelnen Signals zeigte eine praktisch gleiche chemische Verschiebung für die drei Protonen von I an. Dieses Ergebnis stand in Einklang mit der Erklärung der chemischen Verschiebung der geminalen Protonen der endständigen $\mathrm{CH}_{2}-\mathrm{Gruppe}$ in I durch die genannten Autoren, welche die chemische Verschiebung dieser Protonen im Chlor-allen als Referenz verwendeten und nach einer Anisotropie-Korrektur für die Shiftlage der $\mathrm{CH}_{2}$-Gruppe in I einen Wert von $-202 \mathrm{~Hz}$ (relativ zu TMS für $40 \mathrm{MHz}$ ) abschätzten. In beiden Arbeiten konnte keine komplette Analyse des Spektrums durchgeführt werden.

$$
\mathrm{H}_{2} \mathrm{C}=\mathrm{C}=\mathrm{CH}-\mathrm{CN}
$$

Untersucht man jedoch das Kernresonanzspektrum des Cyan-allens in einer etwa 3-proz. Lösung in $\mathrm{CCl}_{4}$ bei einer Meßfrequenz von $100 \mathrm{MHz}$ bei genügend weiter Spreitung (z. B. 250 oder $100 \mathrm{cps}$ sweep-width), so kann man das theoretisch zu erwartende $\mathrm{AB}_{2}$-Spektrum in seiner Gesamtheit beobachten (s. Abb. 1). Nach erfolgter Kalibrierung ${ }^{3}$ ergeben sich für die einzelnen Linien die in Tab. 1 zusammengestellten Werte.

1 G. S. Reddy, L. Mandell u. J. H. Goldstein, J. Amer. chem. Soc. 83, 4729 [1961].

2 H. Walz u. P. Kurtz, Z. Naturforschg. 18 b, 334 [1963].

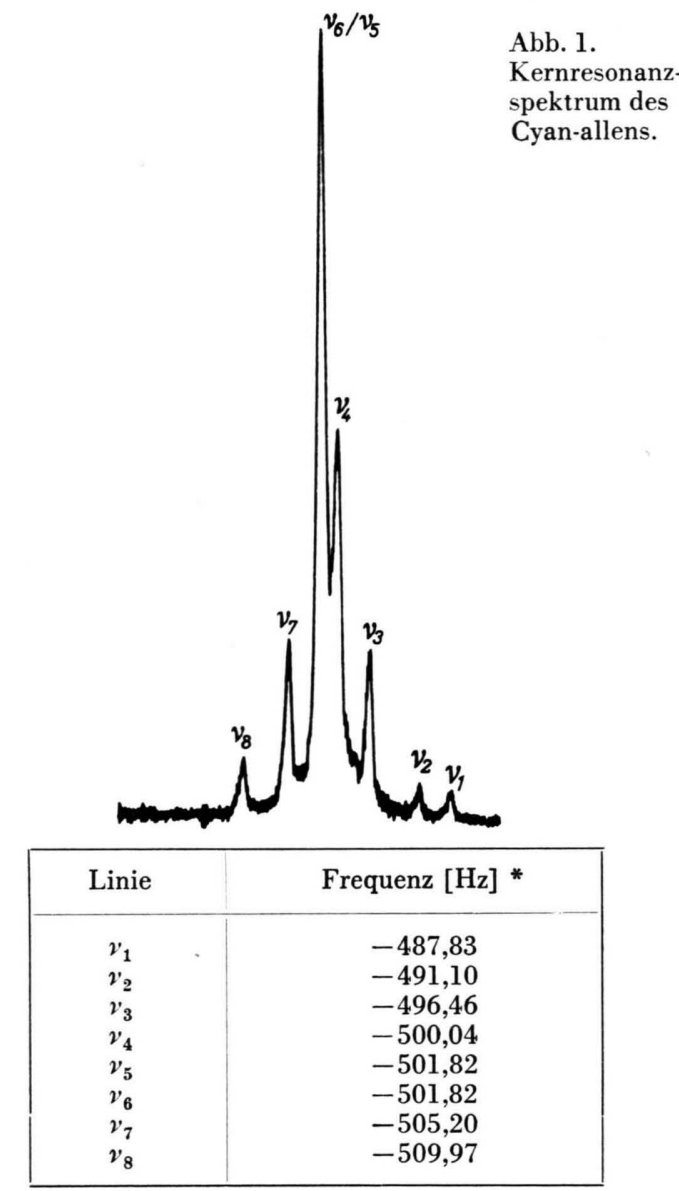

Tab. 1. Vermessene Linien des $\mathrm{AB}_{2}$-Systems des Cyan-allens. * Relativ zu TMS für $100 \mathrm{MHz}$.

${ }^{3}$ Die Kalibrierung erfolgte durch exakte Messung der Frequenz des Frequency-Sweeps an mehreren Stellen des Spektrums unter Benutzung eines Zählers der Fa. HewlettPackard (5245 L Electronic Counter). 
Die Analyse des $\mathrm{AB}_{2}$-Systems liefert unter Verwendung der üblichen expliziten Formeln die entsprechenden Kernresonanz-spektroskopischen Parameter:

$$
\begin{array}{r}
v_{\mathrm{A}}=v_{3}=-496,46 \mathrm{~Hz} \text { von TMS für } 100 \mathrm{MHz}, \\
v_{\mathrm{B}}=\left(v_{5}+v_{7}\right) / 2=-503,51 \mathrm{~Hz} \text { von TMS für } 100 \mathrm{MHz}, \\
\left|J_{\mathrm{AB}}\right|=1 / 3\left[\left(v_{4}-v_{1}\right)+\left(v_{8}-v_{6}\right)\right]=6,79 \mathrm{~Hz} .
\end{array}
$$

Rechnet man die chemischen Verschiebungen auf $40 \mathrm{MHz}$ um, so erhält man für die chemische Verschiebung des $\alpha$-Protons $-198,58 \mathrm{~Hz}$ und für die Shiftlage der geminalen Protonen $-201,40 \mathrm{~Hz}$. Diese Werte sind

4 Vgl. etwa: H. SuHr, in „Anwendungen der kernmagneti. schen Resonanz in der Organischen Chemie" (Organische Chemie in Einzeldarstellungen, Bd. 8), Springer-Verlag, Berlin 1965. dann in sehr guter Übereinstimmung mit den Voraussagen von REDDY und Mitarbb. ${ }^{1}$ sowie von WaLz und Kurtz ${ }^{2}$. Der Wert von $6,79 \mathrm{~Hz}$ für die Kopplungskonstante $J_{\mathrm{AB}}$ liegt in der erwarteten Größenordnung derartiger Kopplungen in Allenen ${ }^{4}$.

Die Messungen wurden am Kernresonanzspektrometer HA-100 der Fa. Varian durchgeführt.

Herrn Dr. P. Kurtz danke ich sehr herzlich für die Überlassung einer Substanzprobe des Cyan-allens, die nach dem von ihm beschriebenen Verfahren ${ }^{5}$ dargestellt wurde. Herrn B. HäHNER danke ich für experimentelle Unterstützung.

5 P. Kurtz, Dtsch. Bundes-Pat. 1064504 v. 1. 2. 1956/3. 9. 1959, Anmeld. Nr. F. 21815 IVb/12021, Farbenfabriken Bayer AG, Leverkusen (C 1960, 6322) ; vgl. auch : P. Kurtz, H. Gold u. H. Disselnkötter, Liebigs Ann. Chem. 624, I [1959].

\section{Die Darstellung der vier möglichen dimeren 1.3-Dimethyluracile durch UV-Bestrahlung von 1.3-Dimethyluracil in einer Eis-Matrix ${ }^{1}$}

\author{
Gerhard Fürst ${ }^{2}$, Egon Fahr und Heinz Wieser \\ Institut für Organische Chemie der Universität Würzburg \\ (Z. Naturforschg. 22 b, 354 [1967] ; eingegangen am 27. Januar 1967)
}

Die bei der UV-Bestrahlung von Nucleinsäuren oder von Pyrimidinen in einer Eis-Matrix (gefrorene wäßrige Lösung) entstehenden dimeren Pyrimidine können in vier isomeren Formen vorliegen. Bisher ist es nicht gelungen, alle vier Isomeren direkt bei der UV-Bestrahlung des Monomeren zu erhalten ${ }^{3}$. Wir konnten nun zeigen, daß bei der UV-Bestrahlung von 1.3-Dimethyluracil in einer Eis-Matrix alle theoretisch möglichen dimeren 1.3-Dimethyluracile gebildet werden.

Eine $1,07 \cdot 10^{-2}-m .^{4}$ wäßrige 1.3-Dimethyluracil-Lösung wurde eingefroren und $40 \mathrm{Min}$. mit UV (SpezialAnalysen-Lampe der Quarzlampen-Gesellschaft, Hanau, mit $\mathrm{Hg}$-Niederdruckbrenner) bestrahlt. Die nach dem Auftauen erhaltenen Bestrahlungsprodukte wurden durch präparative Dünnschichtchromatographie an Kieselgel (Benzol/Aceton 7:3) aufgetrennt. Neben nicht umgesetztem 1.3-Dimethyluracil erhielten wir die vier dimeren Dimethyluracile A, B, C, D im Verhältnis $1: 4: 10: 1,5$ (Tab. 1).

Die Isomeren B, C und D sind thermisch stabil; sie lassen sich analytisch rein darstellen. A dagegen zerfällt bereits beim Stehen bei Raum-Temperatur in 1.3Dimethyluracil, so daß es nicht rein erhalten werden

1 IX. Mitteilung in der Reihe „Die Einwirkung von Strahlung auf Nucleinsäure-Bestandteile“; VIII. Mitt.: E. FAHR, G. Fürst, G. Dörhöfer u. H. Popp, Angew. Chem. im Druck; VII. Mitt.: G. Dörhöfer u. E. FAHR, Tetrahedron Letters [London] 1966, 4511.

2 In Arbeit befindliche Diplomarbeit, Univ. Würzburg. konnte. Bei der Bestrahlung mit kurzwelligem UV in saurer Lösung gehen alle vier Isomeren ausschließlich in 1.3-Dimethyluracil über (dünnschichtchromatographisch überprüft).

\begin{tabular}{|c|c|c|}
\hline Dimeres $^{\mathrm{a}}$ & Schmp. $\left[{ }^{\circ} \mathrm{C}\right]{ }^{\mathrm{b}}$ & $R_{f}$-Wert $\mathrm{c}$ \\
\hline A & $210-220$ & 0,20 \\
B & $245-248$ & 0,24 \\
C & $261-263$ & 0,37 \\
D & $264-266$ & 0,59 \\
d & 123 & 0,49 \\
\hline
\end{tabular}

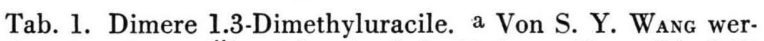
den in einem Übersichts-Artikel, Federat. Proc. 24, S-71 [1965], ohne weitere Angaben zwei dimere 1.3-Dimethyluracile mit den Schmp. $252-253{ }^{\circ} \mathrm{C}$ und $263-264^{\circ} \mathrm{C}$ erwähnt. Vermutlich handelt es sich um unsere Isomeren B und C. b Umkrist. aus Chloroform/Petroläther $\left(40-60^{\circ}\right)$. c Dünnschichtchromatographisch auf Kieselgel (Benzol/Aceton $1: 1$ ). d 1.3-Dimethyluracil.

Obwohl wir zur Zeit keine sichere Zuordnung der vier Isomeren auf die vier möglichen Strukturen vornehmen können, ist doch auf Grund der IR-Spektren und des Vergleichs mit den von Weinblum und Johns ${ }^{3}$ erhaltenen dimeren Thyminen anzunehmen, daß den Isomeren folgende Strukturen zukommen: A cis $(5-6 /$ $6-5), \quad \mathrm{B} \operatorname{cis}(5-5 / 6-6), \quad \mathrm{C}$ trans $(5-5 / 6-6), \mathrm{D}$ trans $(5-6 / 6-5)^{5}$.

Wir danken der Deutschen Forschungsgemeins chaft und dem Fonds der Chemie für die großzügige Förderung der Untersuchungen.

3 Vgl. dazu D. Weinblum u. H. Johns, Biochim. biophysica Acta [Amsterdam] 114, 450 [1966].

4 Verwendet man verdünntere Lösungen, so lassen sich nur die Isomeren $\mathrm{B}$ und $\mathrm{C}$ nachweisen.

5 Zur Nomenclatur s. Lit. ${ }^{1}$. 